\title{
Analyzing Powers of the Deuteron-Proton Breakup in a Wide Phase Space Region
}

\author{
E. Stephan ${ }^{1, a}$, St. Kistryn ${ }^{2}$, N. Kalantar-Nayestanaki ${ }^{3}$, A. Biegun ${ }^{1,3}$, K. Bodek ${ }^{2}$, I. Ciepał ${ }^{2}$, M. Kiš ${ }^{3}$, B. Kłos ${ }^{1}$, A. Kozela ${ }^{4}$, \\ M. Mahjour-Shafiei ${ }^{3, b}$, A. Micherdzińska ${ }^{1, c}$, R. Sworst ${ }^{2}$, J. Zejma² ${ }^{2}$ and W. Zipper ${ }^{1}$ \\ 1 Institute of Physics, University of Silesia, PL-40007 Katowice, Poland \\ 2 Institute of Physics, Jagiellonian University, PL-30059 Kraków, Poland \\ 3 Kernfysisch Versneller Instituut, NL-9747 Groningen, The Netherlands \\ 4 Institute of Nuclear Physics, PAN, PL-31342 Kraków, Poland
}

\begin{abstract}
High precision vector and tensor analyzing powers for the ${ }^{1} H(\mathbf{d}, p p) n$ breakup reaction were measured at $130 \mathrm{MeV}$ beam energy with the detection system covering a large part of the phase space. Results are compared with rigorous theoretical calculations based on realistic nucleon-nucleon potentials, also with so-called three-nucleon force included, as well as on chiral perturbation theory. Theoretical predictions generally describe data quite well although in some regions discrepancies have been observed, what indicates incompleteness of the present-day treatment of the three nucleon system dynamics.
\end{abstract}

\section{Introduction}

Deuteron-proton breakup can serve as a very rich testing ground for modern calculations based on model nucleonnucleon interactions and including also subtle effects of the so-called three-nucleon force (3NF). Due to development of highly polarized beams and accessibility of fast data acquisition systems, it has recently became possible to achieve precision of the many-parameter analyzing power data for this process which is sufficient to enable studies of subleading effects of the $3 \mathrm{~N}$ system dynamics.

A dedicated experiment has been performed at KVI Groningen, with the use of $130 \mathrm{MeV}$ transversaly polarized deuteron beam. Seven different beam polarization states were used, with various combinations of vector and tensor polarizations $\left(P_{Z}, P_{Z Z}\right):(0,0),(2 / 3,0),(-2 / 3,0),(0,1),(0$, $-2),(1 / 3,1),(1 / 3,-1)$. The actual beam polarizations were between $50 \%$ and $80 \%$ of the listed above maximal values. The charged reaction products were registered with a high acceptance position-sensitive detection system SALAD. In the first stage a large set of exclusive, high precision cross section data for the ${ }^{1} H(\mathbf{d}, p p) n$ breakup reaction has been obtained $[1,2]$, supplemented later with an equally rich set of analyzing power data.

Vector $A_{x}, A_{y}$ and tensor $A_{x x}, A_{x y}, A_{y y}$ analyzing powers for the ${ }^{1} H(\mathbf{d}, p p) n$ breakup reaction were determined for a set of 87 angular geometries of outgoing protons covering a significant part of the phase space. Alternatively,

\footnotetext{
a e-mail: elzbieta.stephan@us.edu.pl

b Present address: University of Tehran, Tehran 1439955961, Iran

c Present address: The George Washington University, Washington DC 20052, USA
}

a simplified (integral) analysis has been performed to determine tensor analyzing power in spherical representation, $T_{20}$, for nearly the same set of kinematical configurations [3]. Moreover, as a byproduct of the beam polarization studies, almost complete angular distributions for three analyzing powers $i T_{11}, T_{20}, T_{22}$, for the elastic $d+p$ scattering have been obtained [4]. All the experimentally determined observables are compared to the results of rigorous Faddeev calculations performed with the use of the modern realistic nucleon-nucleon potentials (CD Bonn, AV18, Nijm I and Nijm II) only, referred to in the following by $2 \mathrm{~N}$, as well as including the Tucsone-Melbourne three nucleon force model (2N+TM99) [5]. The experimental data are also confronted with the results of the coupled channel approach with the $\mathrm{CD}-\mathrm{Bonn}+\Delta$ potential, without $(\mathrm{CDB}+\Delta)$ and with $(\mathrm{CDB}+\Delta+\mathrm{Coul})$ Coulomb interaction included [6], as well as with the predictions obtained within the Chiral Perturbation Theory (ChPT) framework - at NNLO including the full dynamics and, currently not complete (without 3NF), N3LO approach [7].

\section{Analysis of elastic scattering}

The procedure used to determine values of the beam polarization relies on analysis of the elastic scattering events. For $\theta_{d}^{C M} \leq 119^{\circ}$ values of the analyzing powers of elastic scattering were determined in a dedicated experiment [8], using absolute calibration of the beam polarization via ${ }^{12} C(\mathbf{d}, \alpha){ }^{10} B^{*}\left[2^{+}\right]$reaction [9]. Knowing vector and tensor analyzing powers at $\theta_{d}^{C M}=119^{\circ}$, values of vector and tensor polarization of the deuteron beam were determined.

On the other hand, knowing the vector and tensor polarizations, almost complete angular distributions for three 


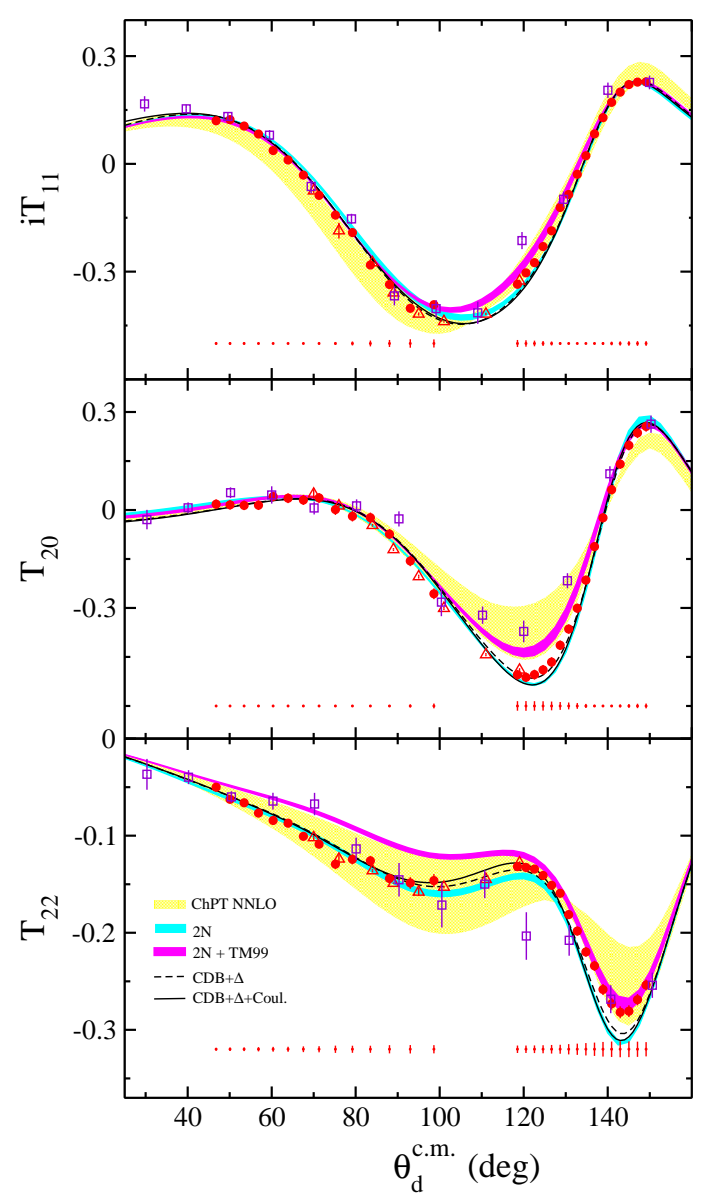

Fig. 1. Vector and tensor analyzing powers for dp elastic scattering at $130 \mathrm{MeV}$ : full dots - this experiment, empty squares - data from [11], triangles - RIKEN data [8]. Theoretical predictions of different approaches are shown as lines and bands, as specified by the legend in the panel. Figure taken from [4].

analyzing powers, $i T_{11}, T_{20}, T_{22}$, for the elastic $d+p$ scattering have been obtained [4]. These distributions are presented in Fig. 1 together with other experimental data, as well as lines and bands representing theoretical predictions. Very clearly, vector analyzing power $i T_{11}$ is well described by all the theories, while tensor analyzing powers reveal certain sensitivity to the applied theoretical approach. The best description of $T_{20}$ is given by calculations with pure $2 \mathrm{~N}$ realistic potentials and also within the coupled-channel framework with the CD Bonn $+\Delta$ potential. Predictions of ChPT and 2N+TM99 calculations differ significantly from the experimental data in the region of $\theta_{d}^{c . m}$. around $120^{\circ}$. The most interesting behavior is revealed by $T_{22}$, for which the largest effects of $3 \mathrm{NF}$ are predicted. For $\theta_{d}^{\text {c.m. }}$ below $120^{\circ}$ the conclusions reached in Ref. [8] are confirmed: pure $2 \mathrm{~N}$ and $\mathrm{CDB}+\Delta$ calculations are consistent with the data. However, at larger $\theta_{d}^{c . m}$. the data points follow the $2 \mathrm{~N}+\mathrm{TM} 99$ predictions. ChPT calculations for $T_{22}$ agree with the data over the whole angular range, but the theoretical uncertainty band is wide. For all studied observables the effects of including Coulomb force into the coupled channel calculations $(\mathrm{CDB}+\Delta+\mathrm{Coul}$.) are negligible.

\section{Analyzing powers for breakup reaction}

In studies of ${ }^{1} H(\mathbf{d}, p p) n$ reaction, performed with transversally polarized beam, some terms of the general formula given in [10] vanish, leading to the following dependence of the cross section on the two vector and three tensor analyzing powers:

$$
\begin{aligned}
& I(\xi, \phi)=I_{o}(\xi) \cdot\{1+ \\
&+ \frac{3}{2} P_{Z}\left[-\sin \phi A_{x}(\xi)+\cos \phi A_{y}(\xi)\right]+ \\
&+ \frac{1}{2} P_{Z Z}\left[\sin ^{2} \phi A_{x x}(\xi)+\cos ^{2} \phi A_{y y}(\xi)+\right. \\
&\left.\left.\quad-\sin 2 \phi A_{x y}(\xi)\right]\right\}
\end{aligned}
$$

where $I_{o}(\xi)$ is the cross section for an unpolarized beam and $\xi$ represents a relevant set of the kinematical variables, $\xi=\left(\theta_{1}, \theta_{2}, \varphi_{12}=\varphi_{2}-\varphi_{1}, S\right)$, i.e. proton emission angles and the variable $S$, denoting the arc-length along the kinematical curve with 0 set at the minimal energy of the second proton. Vector polarization $P_{Z}$ and tensor polarization $P_{Z Z}$ of the beam are both defined with respect to the vertical quantization axis. The coordinate system is defined according to convention of Ref. [10], with $z$ axis along the beam direction and $x$ axis obtained by projection of the momentum of one proton onto the plane perpendicular to $z$. In this system $\phi$ denotes an angle between the $y$ axis and the projection of the spin quantization axis onto the $x y$ plane. $A_{x}(\xi)$ and $A_{y}(\xi)$ are vector analyzing powers while $A_{x x}(\xi), A_{y y}(\xi)$ and $A_{x y}(\xi)$ are tensor analyzing powers, in the Cartesian representation. Formula (1) can be expressed in an equivalent way in spherical coordinates [10]:

$$
\begin{array}{r}
I(\xi, \phi)=I_{0}(\xi) \cdot\left\{1+\sqrt{3} P_{Z} \times\right. \\
\times\left[\sin \phi \operatorname{Im} i T_{11}(\xi)+\cos \phi \operatorname{Re} i T_{11}(\xi)\right]- \\
-\frac{1}{4} P_{Z Z}\left[\sqrt{2} \operatorname{Re} T_{20}(\xi)+\right. \\
\left.\left.+2 \sqrt{3} \cos 2 \phi \operatorname{Re} T_{22}(\xi)+2 \sqrt{3} \sin 2 \phi \operatorname{Im} T_{22}(\xi)\right]\right\} .
\end{array}
$$

In this representation, the analyzing power component $\operatorname{Re} T_{20}$ appears in the term not depending on $\phi$, the only one among all polarization dependent terms which survives after integration of $I(\xi, \phi)$ over the full range of $\phi$ :

$$
\int_{0}^{2 \pi} I(\xi, \phi) d \phi=2 \pi \cdot I_{0}(\xi) \cdot\left[1-\frac{\sqrt{2}}{4} P_{Z Z} \operatorname{Re} T_{20}(\xi)\right]
$$

This features the method which was applied to determine $\operatorname{Re} T_{20}$ (later in this paper referred to as $T_{20}$ ). The measured rates were integrated over the whole range of azimuthal angles, taking advantage of the symmetry of the detection system. Analysis relying on the integrated rates allows one to extract $T_{20}$ independently from all other analyzing power components, thus free of uncertainties due to possible correlations between them in case a fit of expression (1) or (2) to the distribution of rates is used. 

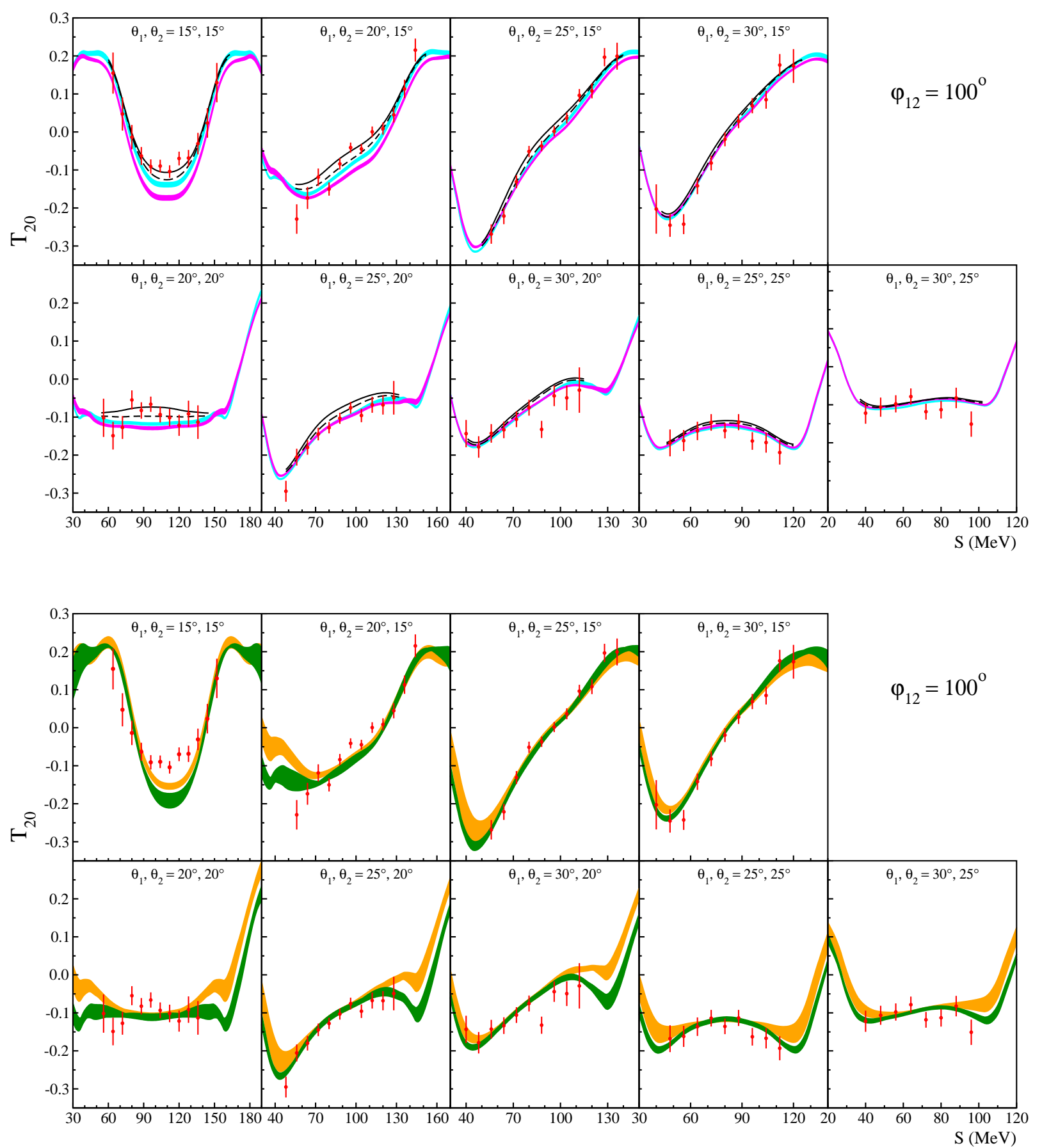

Fig. 2. Breakup analyzing power $T_{20}$ for 9 kinematical configurations characterized by the relative azimuthal angle $\varphi_{12}=100^{\circ}$ and for various combinations of polar angles $\theta_{1}, \theta_{2}$ of the two coincident protons [3]. Error bars reflect only statistical uncertainties. Upper part: The results are compared to predictions obtained with the realistic NN potentials only (cyan bands) and when the TM99 3NF is included into calculations (magenta bands). Theoretical predictions obtained within the coupled-channel framework with the CDBonn $+\Delta$ potential without (dashed line) and with (solid line) Coulomb force are also shown. Lower part: The same data are compared to predictions obtained on the basis of the ChPT approach at NNLO (green bands) and (incomplete) N3LO (orange bands). 


\section{1 $T_{20}$ for breakup reaction}

The tensor analyzing powers $T_{20}(\xi)$ have been obtained as a function of $S$ for 81 angular configurations of the two protons [3]. The geometries have been selected such that $\varphi_{12}$ was taken from $20^{\circ}$ to $180^{\circ}$ with a step of $20^{\circ}$ and all combinations of $\theta_{1}, \theta_{2}$ from the set of $15^{\circ}, 20^{\circ}, 25^{\circ}$ and $30^{\circ}$ were used. Here only a sample of the results, for $\varphi_{12}=100^{\circ}$, is shown in Fig. 2, compared to the predictions of various theoretical approaches. In most of the presented configurations all the theoretical calculations provide very good description of the data. The exception from this rule is observed for geometry characterized with $\theta_{1}=$ $\theta_{2}=15^{\circ}$, where the situation resembles the pattern of (in-)consistencies present in the elastic scattering case: The best description of $T_{20}$ is given by calculations within the coupled-channel framework, while the predictions of ChPT and 2N+TM99 calculations differ strongly from the data. There is, however, also a very small region within the studied kinematical range where all predictions differ from the data significantly [3]. It is localized at low $\varphi_{12}$ and for combinations of the largest studied $\theta_{1}, \theta_{2}$ angles $\left(25^{\circ}\right.$ and $\left.30^{\circ}\right)$.

\subsection{Analyzing powers in Cartesian representation}

The vector $A_{x}, A_{y}$ and tensor $A_{x x}, A_{x y}, A_{y y}$ analyzing powers of the breakup reaction have been studied for a set of kinematical configurations of the two outgoing protons spanned on a grid of azimuthal angles $\theta_{1}, \theta_{2}$ between $15^{\circ}$ and $30^{\circ}$ with the step of $5^{\circ}$ and relative polar angles $\varphi_{12}$ between $0^{\circ}$ and $180^{\circ}$ with the step of $20^{\circ}$. They are obtained in function of arc-length $S$ along the kinematical curve, leading to the total of nearly 1000 data points for each observable. For the purpose to determine these observables, breakup events collected in the given polarization state were sorted according to the set of kinematical variables $\xi$ and the angle $\phi$. After normalization, the numbers of events $N_{P}^{\xi}(\phi)$ for the selected polarization state $P$ were obtained as a function of the angle $\phi$, and a ratio

$$
f_{P}^{\xi}(\phi)=\frac{N_{P}^{\xi}(\phi)-N_{0}^{\xi}(\phi)}{N_{0}^{\xi}(\phi)}
$$

was constructed. $N_{0}^{\xi}(\phi)$ denotes the analogously obtained number of events for the unpolarized beam. Applying the formula (1), $f_{P}^{\xi}$ can be written as:

$$
\begin{array}{r}
f_{P}^{\xi}(\phi)=\kappa\left[1-\frac{3}{2} \sin \phi P_{Z} A_{x}(\xi)+\frac{3}{2} \cos \phi P_{Z} A_{y}(\xi)+\right. \\
+\frac{1}{2} \sin ^{2} \phi P_{Z Z} A_{x x}(\xi)+\frac{1}{2} \cos ^{2} \phi P_{Z Z} A_{y y}(\xi)- \\
\left.-\frac{1}{2} \sin 2 \phi P_{Z Z} A_{x y}(\xi)\right]-1 .
\end{array}
$$

Here $\kappa$ denotes a ratio of normalization factors for the two states, which should be equal to 1 in the case of perfect normalization. The polarizations $P_{Z}$ and $P_{Z Z}$, as well as $\kappa$ values are known from the analysis of the elastic scattering.

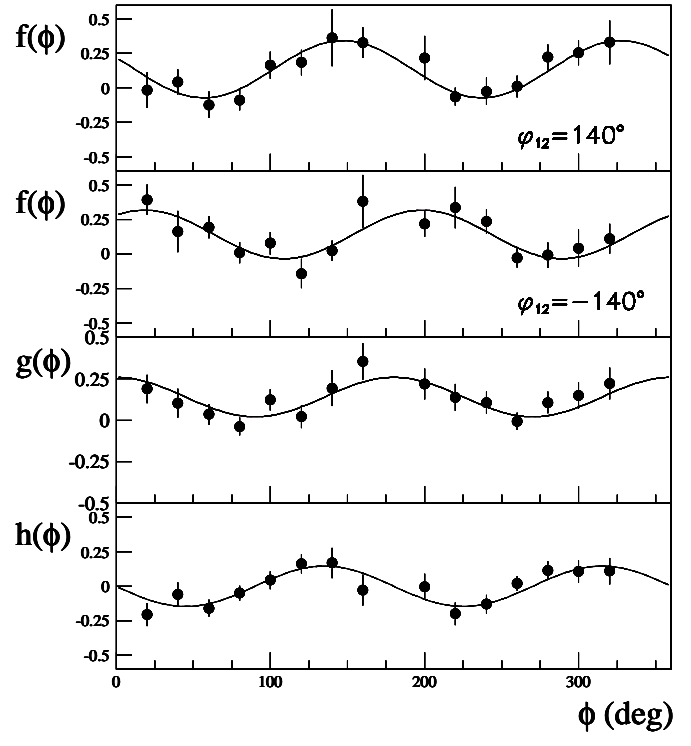

Fig. 3. Example of asymmetry distributions obtained for pure tensor polarization state $\left(\mathrm{P}_{Z}=0, \mathrm{P}_{Z Z}=-2\right)$ and two (mirror) kinematical configurations with $\theta_{1}=25^{\circ}, \theta_{2}=20^{\circ}, \varphi_{12}= \pm 140^{\circ}$ and $S=$ $100 \mathrm{MeV}$. In the two top panels asymmetries $f_{P}^{\xi}(\phi)$ for these configurations are presented; the two bottom panels show their linear combinations, $g_{P}^{\xi}(\phi)$ and $h_{P}^{\xi}(\phi)$. The error bars represent statistical uncertainties. The lines result from the fit of the corresponding functions, as described in the text.

Vector and tensor analyzing powers were extracted from the fit of the function (5) to the experimental distributions of $f_{P}^{\xi}(\phi)$. Example of such distributions and curves fitted to them, obtained for two mirror configurations (i.e. configurations differing only in the sign of $\varphi_{12}$ ), is shown in the two upper panels of Fig. 3. Finally, the weighted averages of the analyzing power values extracted for various polarization states were calculated.

In order to decrease statistical errors of the data points, the results obtained for the kinematical configurations with $\pm \varphi_{12}$ were combined together according to the parity rules:

$$
A_{\alpha}(\xi)=\frac{1}{2}\left[A_{\alpha}\left(\xi^{\prime}, \varphi_{12}\right)+(-1)^{k} A_{\alpha}\left(\xi^{\prime},-\varphi_{12}\right)\right],
$$

where $k=0,1$ for the even $\left(A_{y}, A_{x x}, A_{y y}\right)$ and odd $\left(A_{x}\right.$, $A_{x y}$ ) observables, respectively, and $\xi^{\prime}$ denotes all kinematical variables but $\varphi_{12}$. For the purpose of testing the data consistency, the observables are combined also in the following way:

$$
O_{\alpha}(\xi)=A_{\alpha}\left(\xi^{\prime}, \varphi_{12}\right)-(-1)^{k} A_{\alpha}\left(\xi^{\prime},-\varphi_{12}\right) .
$$

All such combinations should be consistent with 0 within their statistical errors. Obviously, both ways of combining analyzing powers do not apply to the coplanar configurations of the outgoing protons, which transform to themselves with reversing the sign of $\varphi_{12}$.

In the approach described above, which in the following will be further called "free fit method", the constrains set by parity conservation are used only at the very last 


\section{$19^{\text {th }}$ International IUPAP Conference on Few-Body Problems in Physics}

step of analysis, when the analyzing powers obtained for mirror configurations are combined according to Eq. (6). Alternatively, the parity conservation rules have been applied at an earlier stage, before the fit was performed. The appropriate combinations of asymmetries for mirror configurations $\left(\xi^{\prime}, \varphi_{12}\right)$ and $\left(\xi^{\prime},-\varphi_{12}\right)$ have been constructed:

$$
g_{P}^{\xi}(\phi)=\frac{f_{P}^{\xi^{\prime}, \varphi_{12}}(\phi)+f_{P}^{\xi^{\prime},-\varphi_{12}}(\phi)}{2}
$$

and

$$
h_{P}^{\xi}(\phi)=\frac{f_{P}^{\xi^{\prime}, \varphi_{12}}(\phi)-f_{P}^{\xi^{\prime},-\varphi_{12}}(\phi)}{2}
$$

which, using Eq.(5) and rules following from parity conservation, can be expressed as

$$
\begin{aligned}
& g_{P}^{\xi}(\phi)=\kappa\left[1+\frac{3}{2} \cos \phi P_{Z} A_{y}(\xi)+\right. \\
& \left.+\frac{1}{2} \sin ^{2} \phi P_{Z Z} A_{x x}(\xi)+\frac{1}{2} \cos ^{2} \phi P_{Z Z} A_{y y}(\xi)\right]-1
\end{aligned}
$$

and

$$
h_{P}^{\xi}(\phi)=\kappa\left[-\frac{3}{2} \sin \phi P_{Z} A_{x}(\xi)-\frac{1}{2} \sin 2 \phi P_{Z Z} A_{x y}(\xi)\right] .
$$

Fit of the function (10) to the experimentally determined distribution $g_{P}^{\xi}(\phi)$ leads to determination of the parity-even analyzing powers, while the odd ones are obtained from the fit of the function $(11)$ to $h_{P}^{\xi}(\phi)$.

Results of both procedures: the free fit method and the second one, with fits to linear combinations of asymmetries ("parity constrained fit"), should lead to the results consistent with each other. It has been proven to be true within statistical uncertainties. Below, the results of the parity constrained fit are presented.

In the majority of the studied configurations of the breakup reaction all the theoretical predictions for the studied observables agree with each other and describe the data very well. In particular it is true for the vector analyzing powers in the whole studied phase space region. Tensor analyzing powers reveal, however, certain sensitivity to the dynamics utilized in the theoretical calculations. There are configurations in which inclusion of the TM99 3NF improves the description (see an example in the top left panel of Fig. 4). Alas, in several other configurations the calculations including the $3 \mathrm{NF}$ contributions lead to a worse agreement with the experimental data (cf. Fig. 4). In order to express quantitatively the description of the whole data set provided by various models and to identify regions where some effects or problems appear, the values of $\chi^{2}$ per degree of freedom have been calculated. They represent departure of the given theoretical prediction from the experimental data taking into account statistical uncertainty of the data points but neglecting any systematic effects or theoretical uncertainties. Since the energy of the relative motion of the two protons, $E_{r e l}$, was an important variable in analyses of various effects in the cross sections case [1, $2]$, thus the dependence of $\chi^{2} /$ d.o.f. on $E_{r e l}$ has been also studied for the analyzing powers. The results obtained for tensor analyzing powers are shown in Fig. 5. $A_{x y}$ is very well described by pure NN interactions, and on the same level of accuracy by ChPT N3LO and the coupled channel calculations with Coulomb force included. Inclusion of TM99 3NF worsens the agreement, particularly in the domain of relative energies below $15 \mathrm{MeV}$. In the case of $A_{x x}$ and $A_{y y}$ certain discrepancies between the data and the calculations are present for the lowest $E_{\text {rel }}$ values. It is also the place where effects of Coulomb interactions can be observed: calculation with Coulomb force included improves the description of $A_{x y}$ and increases discrepancy at $A_{x x}$, while it has no effect on $A_{y y}$. Outside this region, Coulomb force effects are small and distributed in rather chaotic way.
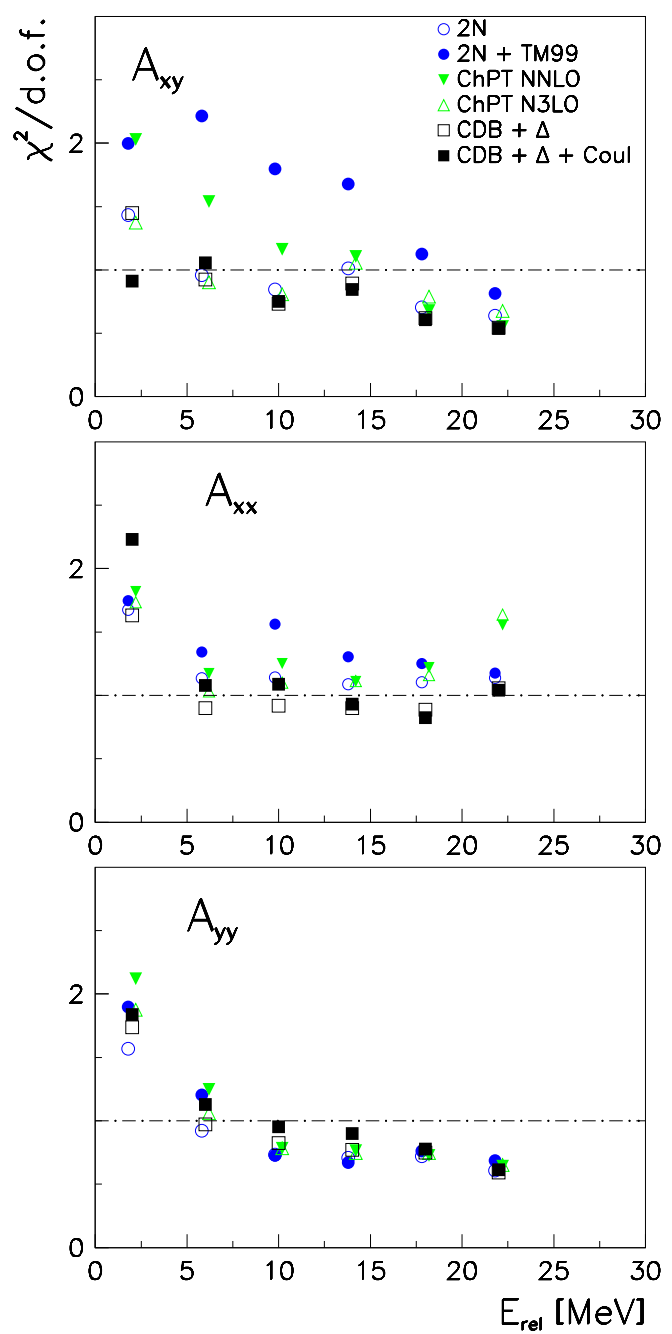

Fig. 5. Quality of description of tensor analyzing powers given by various models presented as $\chi^{2}$ per degree of freedom in function of the relative energy of the two breakup protons.

\section{Summary}

Extensive studies of analyzing powers in the $d p$ system at $130 \mathrm{MeV}$ deuteron beam energy were performed. They 

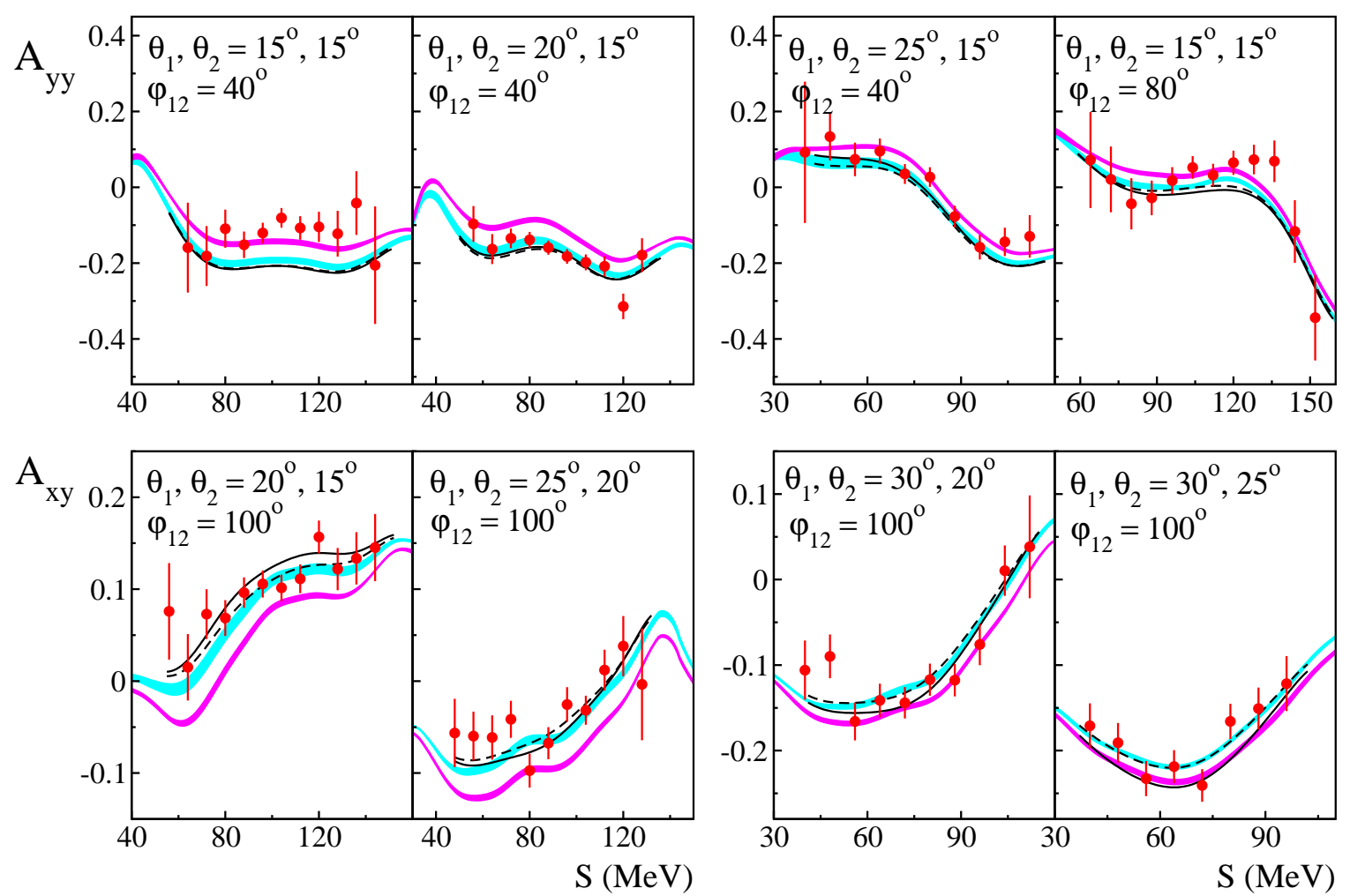

Fig. 4. Examples of tensor analyzing powers results obtained for configurations in which significant effects of the TM99 3 NF are expected. Meaning of bands and lines as in Fig. 2, upper part.

have shown that these observables for the two reaction channels: the elastic scattering and the breakup reaction, reveal certain common features. For example, vector analyzing powers are very well described by the state-of-the-art theoretical calculations and are almost insensitive to the details of the assumed model of dynamics. Tensor analyzing powers, on the other hand, reveal stronger sensitivity to the details of the dynamics, but their precise description in the whole phase space poses a challenge. In spite of a general success, there are kinematical regions where none of the calculations can describe all details of the measured distributions or certain theories provide less adequate description. The tensor analyzing powers $T_{20}$ for the elastic scattering and breakup reactions reveal similar patterns of inconsistencies. Apart from certain similarities between the two channels of the $d p$ reaction, breakup is characterized by a much richer final state kinematics and offers possibility to study observables vanishing in the elastic scattering, like $A_{x}$ and $A_{x y}$. Studies of $A_{x y}$ demonstrate clearly the problem with TM99 3NF. Generally, studies of tensor analyzing powers suggest an existence of some ingredients which are still missing in the spin part of the $3 \mathrm{NF}$ model.

Summarizing, in contrast to the cross section data, which are quite strongly influenced by $3 \mathrm{NF}$ and Coulomb force (in certain regions of phase space), analyzing powers are either insensitive to these pieces of dynamics or, for particular observables and in limited kinematical regions, currently available $3 \mathrm{~N}$ forces seem not to be fully adequate.
This work was partially supported by the Polish 2009 science founds as a research project No. N N202 034836.

\section{References}

1. St. Kistryn et al., Phys. Rev. C 68, 054004 (2003), St. Kistryn et al., Phys. Rev. C 72, 044006 (2005).

2. St. Kistryn et al., Phys. Lett. B 641, 23 (2006).

3. E. Stephan et al., Eur. Phys. J. A 42, 13 (2009)

4. E. Stephan et al., Phys. Rev. C 76, 057001 (2007).

5. W. Glöckle et al., Phys. Rep. 274, 107 (1996).

6. A. Deltuva, A.C. Fonseca, and P.U. Sauer, Phys. Rev. C 72, 054004 (2005),

A. Deltuva, A. C. Fonseca, and P. U. Sauer, Phys. Rev. C 73, 057001 (2006).

7. E. Epelbaum, Prog. Part. Nucl. Phys. 57, 654 (2006).

8. H. Mardanpour et al., Eur. Phys. J. 31, 383 (2007).

9. K. Suda et al., Nucl. Inst. and Meth. in Phys. Res. A 572, 745 (2007).

10. G.G. Ohlsen et al., Nucl. Instr. and Meth. 179, 283 (1981).

11. H. Witała et al., Few-Body Sys. 15, 67 (1993). 